\title{
Le clos du manoir de Kernault à Mellac (Finistère) : des traces d'occupation gauloise aux structurations paysagères de l'Époque moderne
}

The clos of the Kernault manor house in Mellac (Finistère): from traces of Gallic occupation to the landscape structures of the Modern Era

\section{Léa Clouet}

\section{OpenEdition \\ Journals}

Édition électronique

URL : https://journals.openedition.org/rao/6123

DOI : 10.4000/rao.6123

ISSN : 1775-3732

Éditeur

Presses universitaires de Rennes

\section{Édition imprimée}

Date de publication : 18 décembre 2020

Pagination : 191-202

ISBN : 978-2-7535-8232-3

ISSN : 0767-709X

\section{Référence électronique}

Léa Clouet, « Le clos du manoir de Kernault à Mellac (Finistère) : des traces d'occupation gauloise aux structurations paysagères de l'Époque moderne », Revue archéologique de l'Ouest [En ligne], 36 | 2020, mis en ligne le 18 décembre 2020, consulté le 22 août 2022. URL : http://journals.openedition.org/rao/ 6123 ; DOl : https://doi.org/10.4000/rao.6123 


\title{
Le clos du manoir de Kernault à Mellac (Finistère) : des traces d'occupation gauloise aux structurations paysagères de l'Époque moderne
}

\author{
The clos of the Kernault manor house in Mellac (Finistère): \\ from traces of Gallic occupation to the landscape structures of the Modern Era
}

\author{
Léa Clouet $^{\mathrm{a}}$
}

\begin{abstract}
Résumé : L'histoire du manoir de Kernault à Mellac (Finistère) a été récemment enrichie grâce à la réalisation d'un diagnostic archéologique mené par le Centre départemental d'archéologie du Finistère en 2019. La cour et le jardin clos du manoir ont fait l'objet de sondages, offrant ainsi un nouveau regard sur l'histoire du lieu. L'opération a permis de mettre en évidence au moins quatre phases d'occupation. La plus ancienne, caractérisée par la présence d'un probable enclos quadrangulaire, daterait du second âge du Fer. Les vestiges d'une seconde phase d'occupation se perçoivent au travers de la mise en place d'un parcellaire entre la fin $\mathrm{du} \mathrm{XII}^{\mathrm{e}} \mathrm{s}$. et le début du XIII ${ }^{\mathrm{e}}$ s. Cette occupation est abandonnée au profit de la création du manoir et de ses aménagements extérieurs à compter de la fin du $\mathrm{Xv}^{\mathrm{e}} \mathrm{s}$. Une nouvelle structuration spatiale se met en place. La cour du manoir est aménagée et les vestiges reflètent son utilité agricole. Ceint de murs, le jardin clos était structuré par des allées, des haies et des fossés. Trois fosses sont attribuées à une quatrième phase postérieure au XVII ${ }^{\mathrm{e}} \mathrm{s}$.
\end{abstract}

\begin{abstract}
An archaeological evaluation carried out by the Centre Départemental d'Archéologie du Finistère in 2019 as shed new light on the history of the Kernault manor house in Mellac (Finistere). The courtyard and walled garden of the manor house were surveyed, offering a new look at the history of the place. The operation revealed at least four occupation phases. The earliest, represented by the presence of a quadrangular enclosure probably, dates to the Late Iron Age. The delimitation of a parcel of land between the end of the 12th century and the beginning of the 13th century is the second phase of occupation, which subsequently fell out of use when manor house and its annexes were built at the end of the 15th century corresponding to the third phase. The courtyard of the manor house was laid out and the ruins reflect its agricultural use. Alleys, hedges and ditches structured the walled garden. Three ditches were dug during a fourth phase after the 17th century.
\end{abstract}

Mots clés : archéologie des jardins, manoir, fosse d'extraction, fossés, jardin clos, maison forte, second âge du Fer, Moyen Âge, Époque moderne.

Keywords: archaeology of gardens, manor house, extraction pit, ditches, walled garden, fortified house, Late Iron Age, Middle Ages, Modern Era.

\section{Cadre de l'Étude}

\section{Contexte d'intervention}

Le domaine de Kernault, situé sur la commune de Mellac dans le sud du Finistère, à quelques kilomètres de Quimperlé (fig. 1), est un site patrimonial important du Conseil départemental du Finistère. Le manoir et ses jar- dins ont été pour partie classés Monument historique en 1991. Dans le cadre d'un projet d'aménagement paysager des jardins et de réfection du manoir, une étude et un suivi archéologiques ont été demandés par le Département afin d'apporter des clés de réflexion aux restaurateurs. La nature des aménagements ne justifiant pas une fouille préventive, un diagnostic archéologique a néanmoins été prescrit par le service régional de l'archéologie dans l'emprise du clos du

a Archéologue, responsable d’opération, Département de Loire-Atlantique (lea.clouet@orange.fr) 

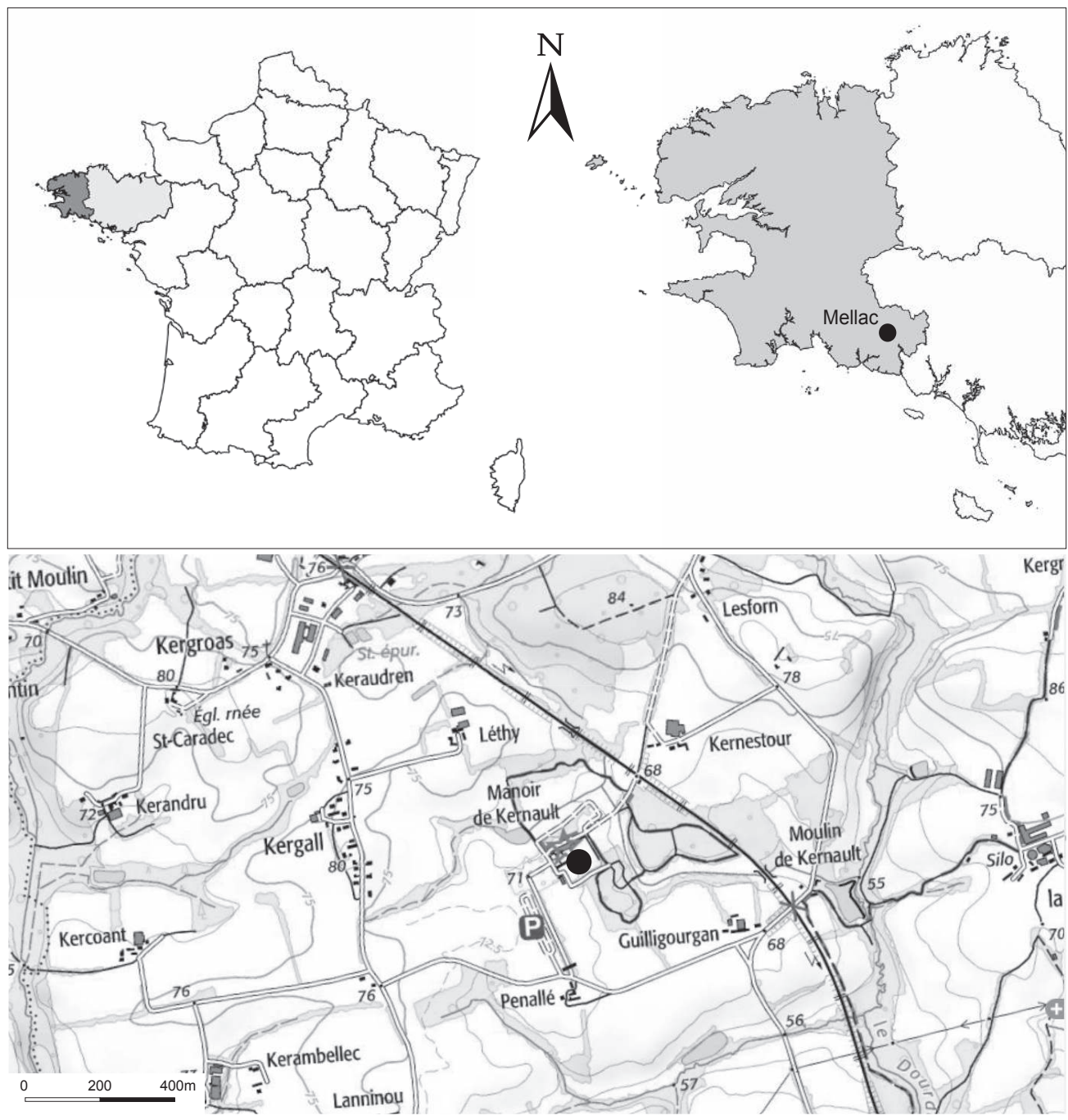

Figure 1 : Localisation de l'opération (DAO L. Clouet, fond de carte [https://cms. geobretagne.fr/]).

Figure 1: Site location (CAD L. Clouet, base map [https:// cms.geobretagne.fr/]). manoir. Le Centre départemental d'archéologie du Finistère est alors intervenu en mars 2019, sondant les $15300 \mathrm{~m}^{2}$ prescrits par le biais de quatorze tranchées, se révélant toutes positives (Clouet, 2019).

\section{Cadre naturel}

Le site est implanté au sud du massif armoricain, sur le domaine varisque méridional sud-armoricain caractérisé, entre autres, par la mise en place de leucogranites au Carbonifère supérieur. Le manoir repose sur un soussol composé de l'un de ces granites : le granite de Baye $(-318+/-7 \mathrm{MA})$. Ce substrat rocheux apparaît par endroits à seulement quelques centimètres sous le sol actuel, lorsqu'il n'est pas recouvert d'arène granitique. La commune de Mellac s'inscrit en contexte de pénéplaine, dans un paysage bocager peu à peu grignoté par l'étalement rurbain. Situé au sud du bourg, sur le bassin versant de l'Isole, le domaine de
Kernault présente une topographie assez plane dont l'altitude avoisine $71 \mathrm{~m} \mathrm{NGF}$

\section{Contexte archéologique}

La carte archéologique ne recense pas moins de trente-cinq entités archéologiques à Mellac, dont les plus anciennes sont attribuées au Mésolithique. À proximité directe du manoir, trois stèles funéraires gauloises sont signalées, une à l'entrée de la cour du manoir, les deux autres à moins de $500 \mathrm{~m}$. Enfin, à moins de $200 \mathrm{~m}$ au nord du manoir subsiste une butte de terre rectangulaire (fig. 2). En 1877, elle fut décrite par E. Flagelle comme un « castel rectangulaire de vingt-huit mètres sur quatorze mètres, avec revêtement en pierres, d'un mètre cinquante de hauteur, entouré d'une douve " (Flagelle, 1878, p. 36). Cette plate-forme est interprétée comme une potentielle maison forte antérieure au manoir de Kernault (Kernévez, 1997, p. 115), caractéristique des résidences de 
petits vassaux au bas Moyen Âge. Aucun élément ne permet de confirmer cette interprétation basée sur des critères morphologiques.

\section{LE MANOIR : PRÉSENTATION DU LIEU}

\section{Historique des recherches}

Les recherches sur l'histoire du manoir furent amorcées à la suite de l'achat du domaine de Kernault par le conseil général du Finistère en 1990 (Pla et Le Rest, 1991; Wanner, 1993; Mignot et Chatenet, 1993). En 1993, l'Inventaire général du patrimoine recense l'ensemble des bâtiments qui composent le manoir ainsi que son domaine
(29 ha aujourd'hui). Une monographie détaillée, adossée au dépouillement des archives, a été réalisée par Jacqueline Le Calvé (1995a). Le rapport dactylographié a fourni la matière à un article (Le Calvé, 1995b).

\section{Histoire du lieu}

La reprise économique qui suivit la guerre de Succession de Bretagne, à la fin du XIv e s., permit la mise en chantier de très nombreux manoirs. Dans la châtellenie de Quimperlé, soixante-deux manoirs furent bâtis entre 1440 et 1499 dont au moins cinq à Mellac (Le Calvé, 1995a, p. 6). Un demisiècle plus tard, une dizaine sont attestés sur la commune (Douard, 2002). Seul celui de Kernault a perduré jusqu’à aujourd'hui. Dès la première moitié $\mathrm{du}_{\mathrm{Xv}} \mathrm{e}^{\mathrm{s}}$., les terres de

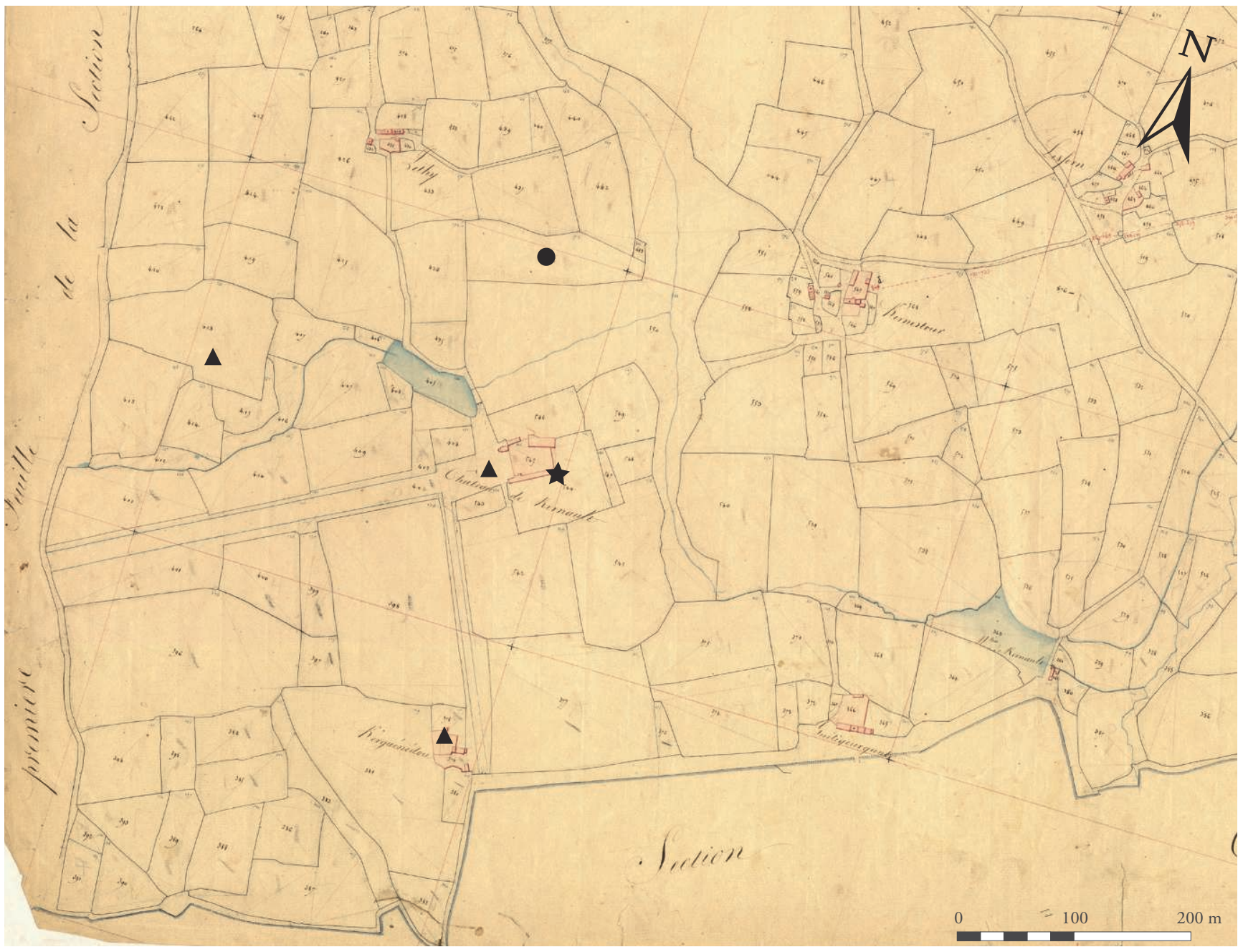

Figure 2 : Localisation du manoir (étoile), des stèles protohistoriques (triangles) et de la plate-forme de terre potentiellement médiévale (rond) sur le cadastre de 1825 (DAO L. Clouet [https://mnesys-portail.archives-finistere.fr]).

Figure 2: Location of the manor house (star), protohistoric stelae (triangles) and the potentially medieval earth platform (round) on the 1825 land register (CAD L. Clouet, [https://mnesys-portail.archives-finistere.fr]). 
Kernault sont détenues par Yvon de Lescoët qui obtient des lettres de noblesse en 1464 avant d'être fait premier seigneur de Kernault en 1470 (Postic, 1994). La construction du logis date vraisemblablement de cette seconde moitié du $\mathrm{XV}^{\mathrm{e}}$ s. $\mathrm{Au} \mathrm{XvI} \mathrm{X}^{\mathrm{e}}$ s., alors que le domaine était la propriété de la famille Le Beuff, plusieurs agrandissements furent réalisés : édification de la chapelle, de la maison du four et de la partie est des communs (Douard, 2000). Au XviI ${ }^{e}$ s., la famille Le Veyer, propriétaire du domaine, entreprit de nombreuses constructions. L'espace intérieur du logis est amélioré en 1627 et, dans la première moitié du XviI ${ }^{\mathrm{e}}$ s., un vaste grenier, avec façade sur cour à pans de bois et corps de passage, fut construit. Cet élément architectural, dont peu d'autres exemples subsistent aujourd'hui en Bretagne, témoigne de l'intense production céréalière de la seigneurie à cette époque (Digou et Le Gal, 2008). Au milieu du XviII ${ }^{\mathrm{e}}$ s., la famille du Vergier de Kerhorlay souhaitant améliorer le confort du manoir commanda un projet d'aménagement à l'architecte Antoine Le Forestier. Les fragments d'un plan de jardin à la française ont été retrouvés dans les archives du manoir, probablement en lien avec le projet proposé par l'architecte. Les transformations planifiées ne seront que très partiellement réalisées : agrandissement des baies, percement d'un passage entre l'entrée et l'office, mise en place des lambris de la grande salle et cloisonnements à l'étage. Après avoir servi de refuge aux contre-révolutionnaires à la fin du $\mathrm{XVIII}^{\mathrm{e}}$ s., le manoir servit de "ferme expérimentale " au cours du XIX ${ }^{e}$ s. Son propriétaire testa de nouvelles techniques agricoles dans son potager (Postic, 1993) et reboisa une partie du domaine. Les murs et grilles de l'entrée du clos datent de cette période. À la fin du XIX ${ }^{\mathrm{e}}$ s., la partie nord du logis fut remaniée et plusieurs bâtiments furent également ajoutés : la longère et la partie ouest des communs. Au cours du Xx s. le domaine resta dans la famille Poulpiquet de Brescanvel (par mariage avec du Vergier). Les dernières modifications furent la construction d'un bûcher et le déplacement des lucarnes du grenier sur le logis.

\section{Le manoir aujourd'hui et la problématique de recherche}

De 1992 à 1998, Kernault bénéficia d'une importante campagne de restauration. Le domaine, géré depuis 2006 par l'EPCC Chemin du Patrimoine en Finistère, fait l'objet d'un nouveau projet dont l'aménagement paysager est au cœur. Dans ce contexte, la première opération archéologique est réalisée en 2019 dans le clos. Les abords d'un manoir, en particulier l'espace clos, le jardin privé, sont de manière générale assez mal connus puisque majoritairement structurés par des aménagements peu pérennes. Du fait d'enjeux touristiques et écologiques, la question des jardins patri- moniaux se développe néanmoins (Gaugain et al., 2019). À Kernault, les investigations archéologiques au service de projets paysagers offrent l'occasion d'apporter un éclairage sur la façon dont s'organisait le pourpris.

\section{LES OBSERVATIONS ARCHÉOLOGIQUES}

L'analyse des vestiges archéologiques a permis d'identifier au moins quatre phases d'aménagements (fig. 3). Notons qu'une trentaine de faits archéologiques n'ont pas pu être attribués à l'une de ces phases, par manque d'élément datant, de recoupement stratigraphique ou de caractéristique particulière. Il est impossible d'émettre des hypothèses interprétatives quant à la fonction de ces structures non phasées. Les trous de poteau peuvent refléter la présence d'aménagements de type clôture ou bâtiment, mais aucun plan ne se dessine et aucun rattachement chronologique n'est possible. Néanmoins, ces faits archéologiques reflètent une certaine densité d'occupation.

\section{Phase I : une occupation du second âge du Fer}

Au vu des vestiges mis au jour et de l'analyse des comblements des fossés, la phase I est caractérisée par l'angle d'un probable enclos fossoyé quadrangulaire dont une entrée a partiellement été observée (fig. 3). Aucun aménagement interne n'a pu être identifié (fig. 4). Sondés mécaniquement, les fossés ont dévoilé des caractéristiques similaires. Les profils de creusements apparaissent en " $V$ » (fig. 5), conservés sur une profondeur moyenne de $0,9 \mathrm{~m}$ et les dynamiques de comblement sont très proches. Le fossé du côté nord

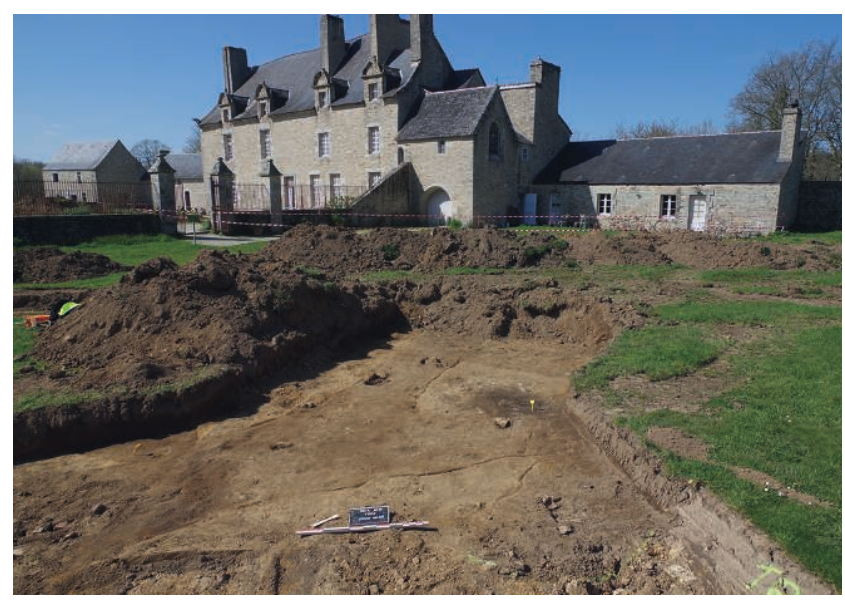

Figure 3 : Recoupement de structures dans la tranchée TR 04, antérieures au manoir (cliché L. Clouet).

Figure 3: Cross-section of features in trench TR 04, earlier than the manor house (photo L. Clouet). 




Figure 4 : Plan des vestiges de la phase I (topographie B. Grall; DAO L. Clouet). Figure 4: Plan of the features of phase I (survey B. Grall; CAD L. Clouet).

est doublé d'un second fossé, plus petit, avec un probable talus central. De rares traces relevées dans TR 07 suivent la même orientation et sont également associées à cette première phase d'occupation. Le mobilier retrouvé dans l'un des fossés ainsi qu'une datation ${ }^{14} \mathrm{C}$ attestent d'une occupa- tion située autour des $\mathrm{IV}^{\mathrm{e}}-\mathrm{III}{ }^{\mathrm{e}}$ s. av. n. è. En l'état actuel des connaissances, il est impossible d'affirmer la vocation et le statut de ce site. La question du lien entre ce potentiel enclos et les stèles funéraires situées à proximité directe se pose et demande à être explorée. 


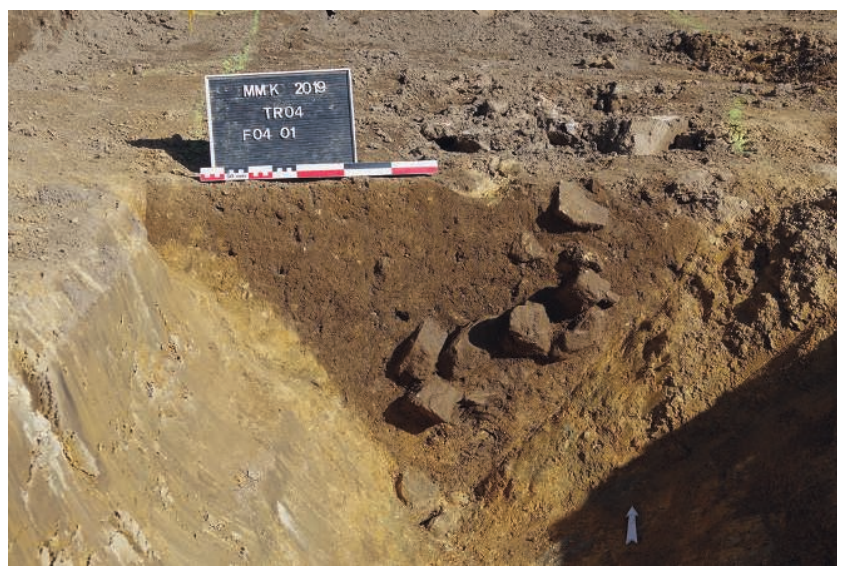

Figure 5 : Vue en coupe du fossé F 04.01 (cliché L. Clouet). Figure 5: Section of the ditch F 04.01 (photo L. Clouet).

\section{Phase II : une occupation entre la fin du XII ${ }^{e}$ et le $X V^{e} s$.}

Un ensemble de structures fossoyées forme une nouvelle trame postérieure aux structures de la phase I. La structuration orthonormée de la phase II, orientée NNO/SSE-NNE/ SSO n'est axée ni sur les vestiges de la phase I, ni sur les aménagements manoriaux (fig. 6). Les données archéologiques permettent de situer la phase II dans une fourchette de temps comprise entre la fin du $\mathrm{XII}^{\mathrm{e}}$ s. ou au début du XIII ${ }^{\mathrm{e}}$ s. au plus tôt, et la construction du manoir au $\mathrm{Xv}^{\mathrm{e}} \mathrm{s}$. Il s'agit vraisemblablement d'une forme de parcellaire, sans indice spécifique concernant le type d'occupation.

\section{Phase III : entre la fin du $\mathrm{XV}^{\mathrm{e}}$ et le début du XVII ${ }^{\mathrm{e}}$ s. : les aménagements extérieurs du manoir}

Le manoir, édifié dans la seconde moitié du $\mathrm{Xv}^{\mathrm{e}}$ s., se caractérise par un pourpris quadrangulaire clos de murs dont l'angle nord-est est occupé par la cour. Une grande allée rejoint la grille d'accès au manoir à l'ouest. Le logis n'a pas été édifié dans l'axe de l'entrée mais au nord de la cour, faisant face aux bâtiments à vocation agricole. Létude des vestiges archéologiques liés à cette phase (fig. 7) permet d'appréhender l'organisation spatiale et la vocation des aménagements extérieurs, de la cour et du jardin (fig. 8). La contemporanéité de l'ensemble des vestiges de la phase III n'est pas confirmée et les éléments datant sont rares. Le début de la mise en place de ces structures, liées à la construction du manoir, a dû être réalisée à compter de la seconde moitié du Xv ${ }^{e}$ s. ou au début du XVI $I^{e}$. La disparition de ces aménagements a dû s'opérer en plusieurs temps. Nous constatons en effet des vestiges postérieurs (phase IV) dont les éléments de datation permettent de les placer au Xvi ${ }^{\mathrm{e}}$ ou
$\mathrm{XVII}^{\mathrm{e}} \mathrm{s}$. (un fragment d'albarelle en grès normand ainsi qu'un fragment de bord d'un pot), et constatons également que d'autres structures ont perduré dans le temps, comme cela a pu être observé sur des clichés du $\mathrm{XIX}^{\mathrm{e}}$ ou $\mathrm{XX}^{\mathrm{e}} \mathrm{s}$.

\section{La cour}

Sous les allées et les parterres végétalisés actuels de la cour du manoir (fig. 9), les vestiges offrent une vision différente de ce à quoi pouvait ressembler cet espace entre le XvI et le début du $\mathrm{xx}^{\mathrm{e}} \mathrm{s}$. Des aménagements de sols ont été identifiés dans la tranchée TR 14. Deux zones distinctes ont été empierrées : au nord, une zone potentiellement ovale et au sud, l'amorce d'une zone relativement similaire. Ces empierrements ont certainement eu pour fonction d'apporter une stabilité au sol et de favoriser le drainage des eaux de pluie. L'unique bordure bien agencée avec des grandes dalles plates qui a pu être observée se situe en zone périphérique de la cour (US 14.01). À l'est, les vestiges d'un aménagement en bois ont été exhumés. Des trous d'ancrage de poteaux (F 13.05 et F 13.06) sont associés à des fossés d'accès sur la façade du logis. Il est possible que les fossés aient assuré une fonction drainante, d'autant plus nécessaire que l'ouest de la cour n'est pas empierré.

D'après ce qui a pu être observé dans la cour, on constate une partition de l'espace. Les axes de circulation sont potentiellement des marqueurs de cette organisation spatiale. D'une part, l'axe NO/SE dans le prolongement de la porte du grenier à pans de bois semble créer une délimitation entre l'empierrement F 14.01 et les vestiges exhumés dans la tranchée TR 13. D'autre part, l'interruption des niveaux empierrés F 14.01 et F 14.02 se situe exactement dans l'axe NE/SO formé par le portail ouest et le portail est donnant sur le jardin. Les vestiges mis en évidence reflètent une cour à usage agricole, dont l'entrée principale située à l'ouest aurait bénéficié d'aménagement spécifique pour la stabilité et le drainage du sol.

\section{Les aménagements paysagers}

Dans le jardin, une structuration paysagère se dessine. Laxe de circulation NO/SE, connu aujourd'hui par la présence d'une porte entre le préau du grenier à pans de bois et le jardin, est marqué par la présence d'une structure de plantation (1 sur fig. 8).

La zone est du jardin est délimitée par un fossé orienté $\mathrm{SO} / \mathrm{NE}$, dans le prolongement du grenier à pans de bois (F 04.04, F 12.04, F 02.09). Des trous de poteau accolés au nord du fossé peuvent suggérer la présence d'un aménagement palissadé. Cet axe scinde le jardin côté est en deux ( 2 au sud et 3 au nord sur fig. 8). Les vestiges situés au sud se caractérisent par la présence de nombreuses fosses de plantation (fig. 10), formant un alignement SO/NE dans la tranchée TR 09. Des éléments structurants sont également 


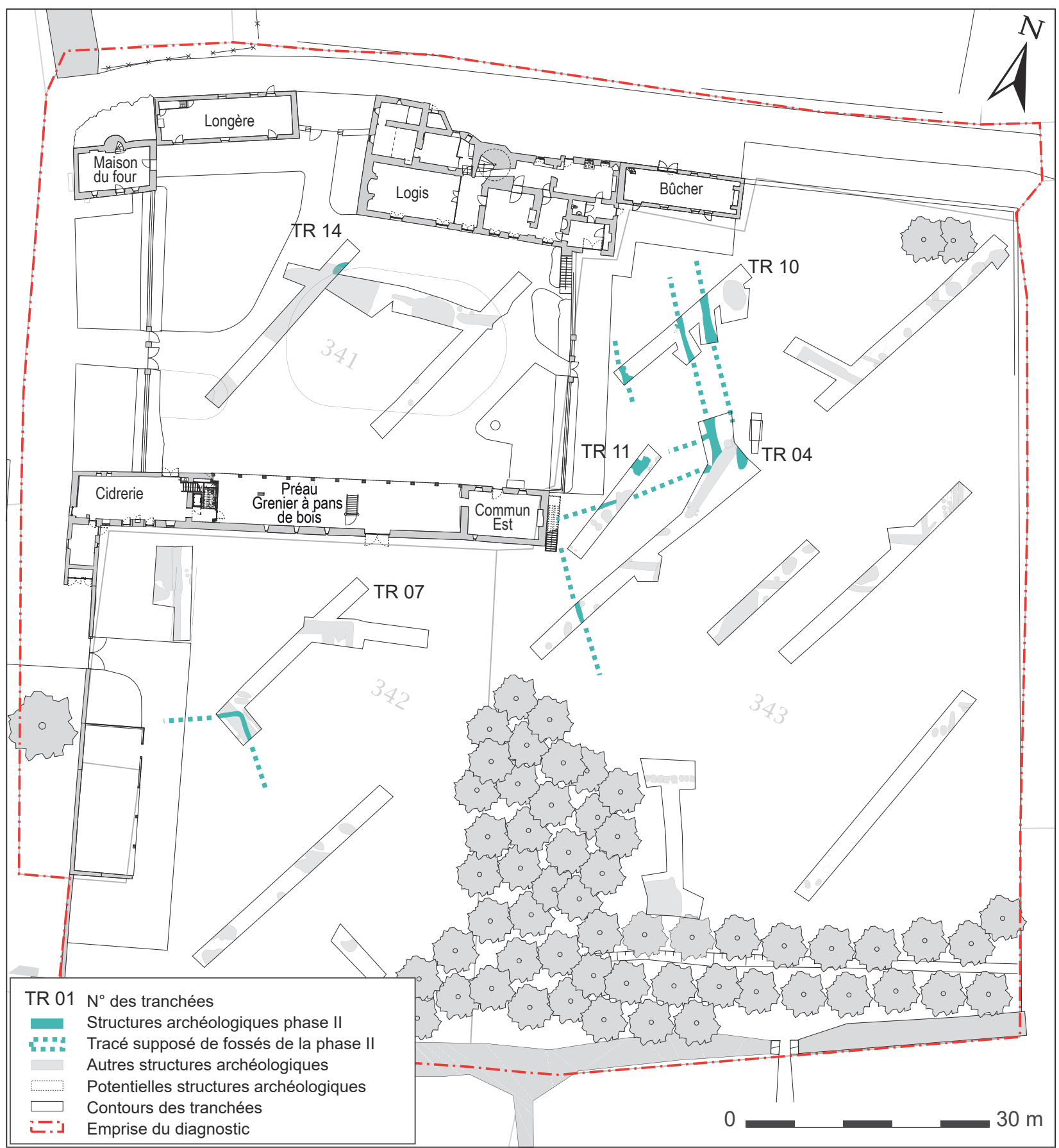

Figure 6 : Plan des vestiges de la phase II (topographie B. Grall; DAO L. Clouet). Figure 6: Plan of the features of phase II (survey B. Grall; CAD L. Clouet).

présents dans la tranchée TR 01. Des probables fosses de plantation sont installées parallèlement aux fosses de la tranchée TR 09, $6 \mathrm{~m}$ au nord (2' sur fig. 8). Deux petits fossés perpendiculaires délimitent également un espace de $7,5 \mathrm{~m}$ de largeur le long du mur du clos. (2" sur fig. 8).

Au sud des plantations de TR 09, les aménagements sont moins nombreux (1'sur fig. 8) et plusieurs chablis ont été repérés. Une grande fosse probablement dédiée à l'extraction de matériaux a également été mise en évidence au sud de cet axe (cf. infra). L'alignement des fosses de plantation dans la tranchée TR 09 pourrait ainsi délimiter un jardin structuré au nord d'un espace plus boisé au sud.

Au nord du fossé structurant (F 04.04, F 12.04, F 02.09), des fossés et fosses de plantation organisent l'espace $\mathrm{n}^{\circ} 3$ 


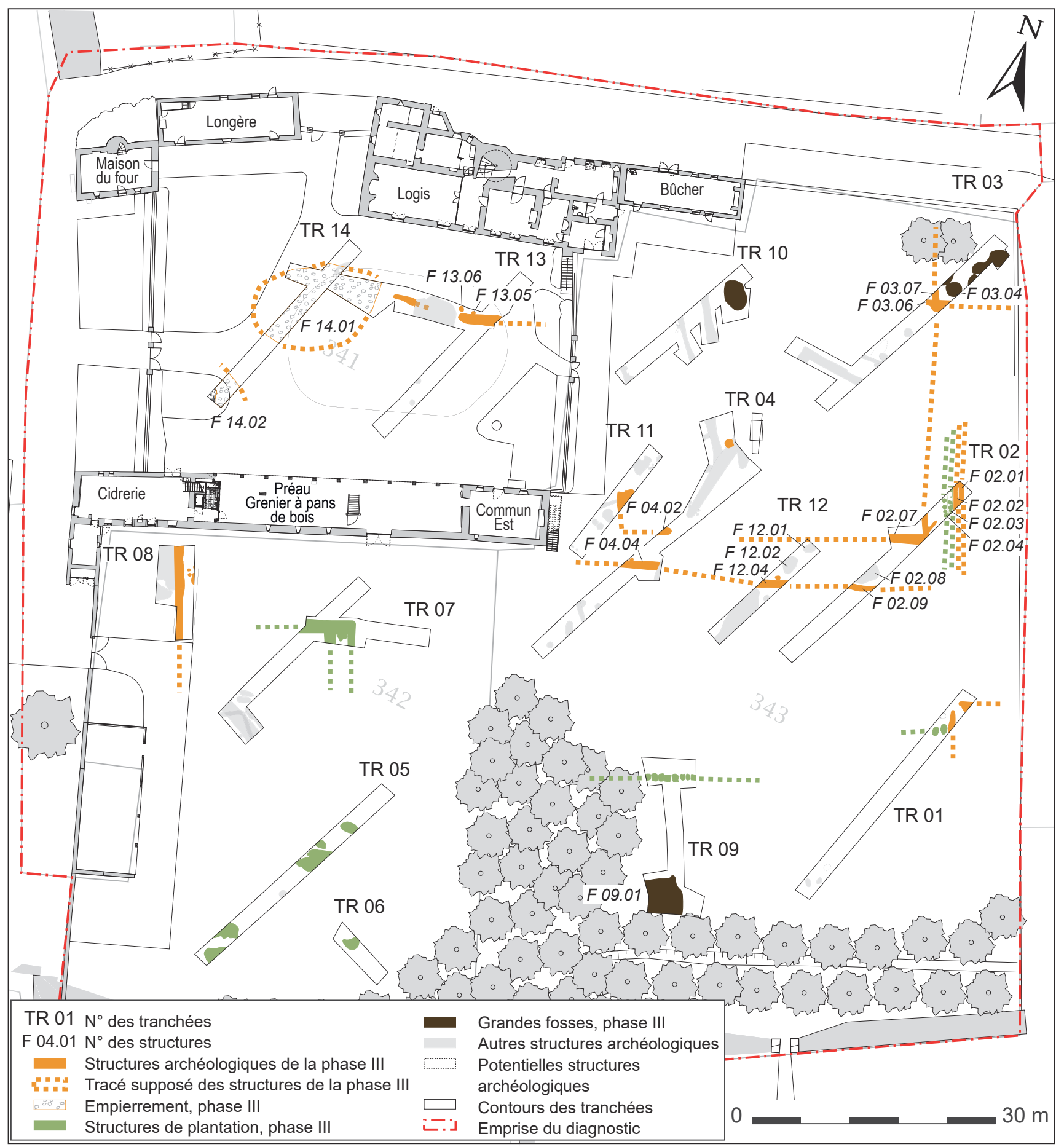

Figure 7 : Plan des vestiges de la phase III (topographie B. Grall; DAO L. Clouet).

Figure 7: Plan of the features of phase III (survey B. Grall; CAD L. Clouet).

(fig. 8). Un axe parallèle fossoyé se dessine à environ $4,5 \mathrm{~m}$ au nord (F 04.02 et F 02.07). L'espace ainsi formé (3' sur fig. 8) a probablement accueilli des plantations puisque des fosses ont été mises au jour (F 12.01, F 12.02, F 02.08). Cependant, il n'est pas possible d'affirmer que ces dernières sont attribuées à la phase III et peuvent tout aussi bien être liées à des aménagements antérieurs ou postérieurs.

Comme le dévoile une photographie issue des archives du manoir, la bande située le long du côté est du clos, large de $7,5 \mathrm{~m}$, a accueilli une rangée de végétaux le long du mur. 


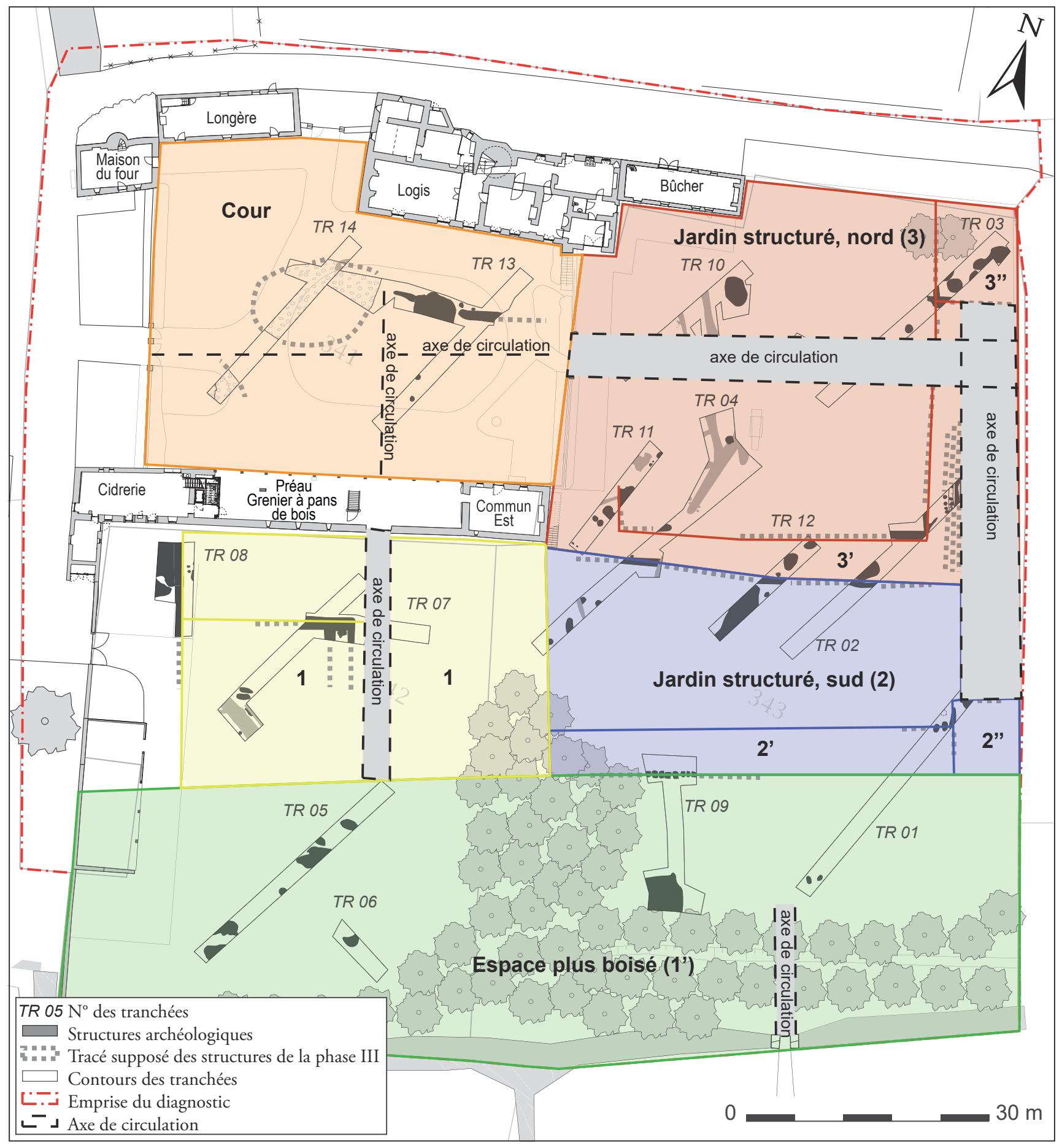

Figure 8 : Schématisation de la structuration spatiale du jardin entre la fin du Xve s. et le XviI s. (topographie B. Grall; DAO L. Clouet). Figure 8: Diagram of the garden's organisation end of 15th-17th century (survey B. Grall; CAD L. Clouet).

L’image montre une allée gravillonnée, qui n’a laissé aucune trace dans le sous-sol, bordée par des plantations. Les faits F 02.01, F 02.02, F 02.03 et F 02.04 en sont les vestiges. D'après la photographie, l'allée se prolongeait vers le nord. Une autre allée, perpendiculaire à cette dernière et plus large, faisait la jonction entre la cour et le portail encore visible aujourd'hui dans le mur est. De même, cette allée n'a laissé aucune trace dans le sous-sol. 


\section{Des fosses d'extractions?}

Enfin, un petit espace carré apparaît à l'angle nord du clos (3" sur fig. 8), fermé par les deux fossés perpendiculaires F 03.06 et F 03.07. À l'intérieur de cet espace, une batterie de grosses fosses circulaires " en grappe " a été identifiée. Le seul plan complet observé est celui de F 03.04 dont le diamètre atteint près de $2 \mathrm{~m}$. Le profil entier observé en coupe atteint une profondeur de $0,9 \mathrm{~m}$. Toutes les fosses sont comblées par de nombreux blocs de granite de tailles variées et de rares fragments de schiste ardoisier mêlé à un limon brun sableux. Deux autres grandes fosses ont été repérées dans le jardin. L'une se situe à $25 \mathrm{~m}$ au $\mathrm{SO}$ des fosses " en grappe ». Le creusement ovale mesure 3,9 m sur 2,7 m et sa profondeur est conservée sur 0,8 m. Le comblement est sensiblement identique à celui des fosses " en grappe ». L'autre fosse, évoquée plus haut, se situe au sud du jardin, dans l'espace «boisé ». Le plan et le profil de la structure n'ont pas été dégagés intégralement mais la fosse a été taillée de manière nette dans la roche granitique sur plus d'1,4 $\mathrm{m}$ de profondeur. Ces structures n'ont livré quasiment aucun mobilier et n'ont donc pas servi de dépotoir. L'hypothèse de fosses d'extraction de matériaux est tout à fait envisageable. Seule la fosse F 09.01 a entamé le substrat granitique propice à la construction de murs. L'argile et le sable ont pu également être recherchés par le creusement des autres fosses. On peut donc évoquer l'hypothèse, qui ne saurait être confirmée que par une étude de bâti et une analyse pétrographique, que des matériaux locaux extraits directement sur place aient participé à la construction du manoir. Le comblement rapide et massif de ces fosses s'expliquerait par le fait qu'une fois la matière première extraite pour un chantier de construction, l'aménagement des jardins fut précédé d'un remblaiement de ces excavations et un nivellement du sol.

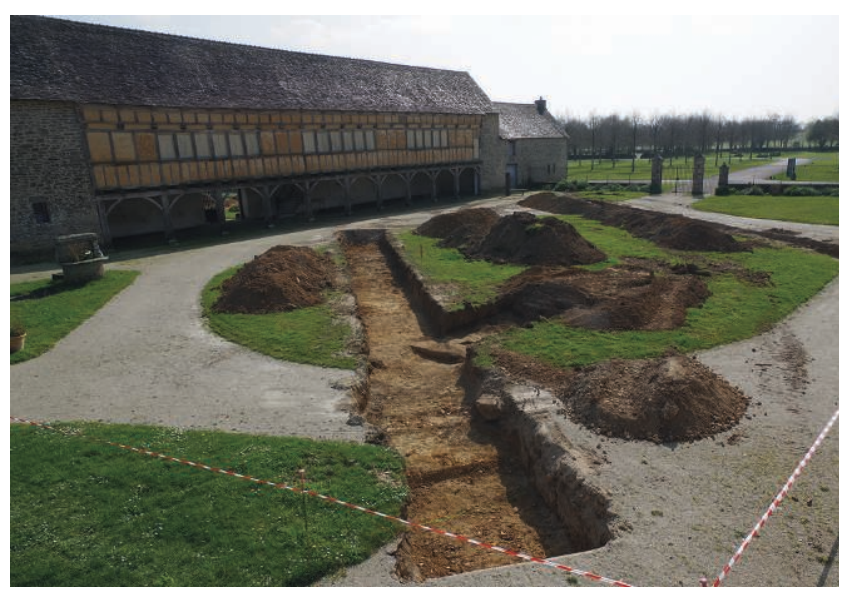

Figure 9 : Vue générale de la cour du manoir, après l'ouverture des tranchées (cliché L. Clouet).

Figure 9: General view of the manor's courtyard, after the opening of the trenches (photo L. Clouet).

\section{Phase IV}

Trois grandes fosses ont été repérées dans les tranchées TR 08 et TR 13 et se sont avérées postérieures à des aménagements attribués à la phase III. Aucune d'entre elles n'a pu être décapée intégralement. Le mobilier issu du comblement supérieur de la fosse F 08.01 est attribué au XVI-XVII ${ }^{\mathrm{e}}$ s., ce qui coïncide avec le mobilier issu des niveaux scellant l'empierrement de la cour. La contemporanéité des trois fosses n'est pas avérée, mais elles semblent toutes trois postérieures à des aménagements extérieurs du manoir. L'état actuel des connaissances ne permet pas de connaitre les fonctions de ces fosses.

\section{Conclusion}

Les résultats du diagnostic mettent en évidence plusieurs phases d'occupation, dont la première remonterait au second âge du Fer. Un hiatus chronologique de près de mille six cents ans sépare la première de la deuxième phase. En effet, ce n'est qu'à la fin du XII ${ }^{e}$ ou au début du XIII ${ }^{\mathrm{e}}$ s. que les vestiges d'une nouvelle occupation apparaissent, caractérisés par la mise en place d'un parcellaire. Il reflète une probable exploitation des terres de Kernault, peut-être en relation avec la plate-forme de terre située à $200 \mathrm{~m}$ au nord du manoir et communément interprétée comme étant la trace d'une maison forte du XIII ${ }^{\mathrm{e}}$ s. Peut-on y voir une continuité d'un lieu de pouvoir, avec déplacement et évolution de l'habitat? Il est assez fréquent qu'un manoir ne soit pas créé ex nibilo mais qu'il succède à une occupation élitaire remontant aux XI-XIII ${ }^{\mathrm{e}} \mathrm{s}$. de type manoir à motte ou maison forte (Brand'Honneur, 2001). Dans son étude sur le Finistère, Patrick Kernévez indique que sur les quatrevingt-onze mottes certaines recensées, cinquante-deux sont associées à un manoir ou à l'existence d'un lignage portant le nom du site d'implantation (Kernévez, 1996, p. 21-24). À Kernault, seules des investigations plus poussées permettraient d'identifier cette plate-forme et de saisir son lien avec le manoir.

L'occupation attribuée à la phase II est abandonnée au profit de la création du manoir et de ses aménagements extérieurs à compter de la fin $\mathrm{du} \mathrm{Xv}^{\mathrm{e}}$ s. Tout d'abord, la mise au jour de fosses probablement dédiées à l'extraction de matériaux peut laisser penser qu'une partie des matières premières nécessaires à la construction ou aux réfections $\mathrm{du}$ manoir a été récupérée directement in situ. D'autre part, les vestiges exhumés dans la cour et le jardin reflètent la bivalence des fonctions du manoir. La fonction agricole s'observe au travers d'aménagements caractéristiques d'une cour de ferme, utilisée, piétinée, sans attributs décoratifs spé- 


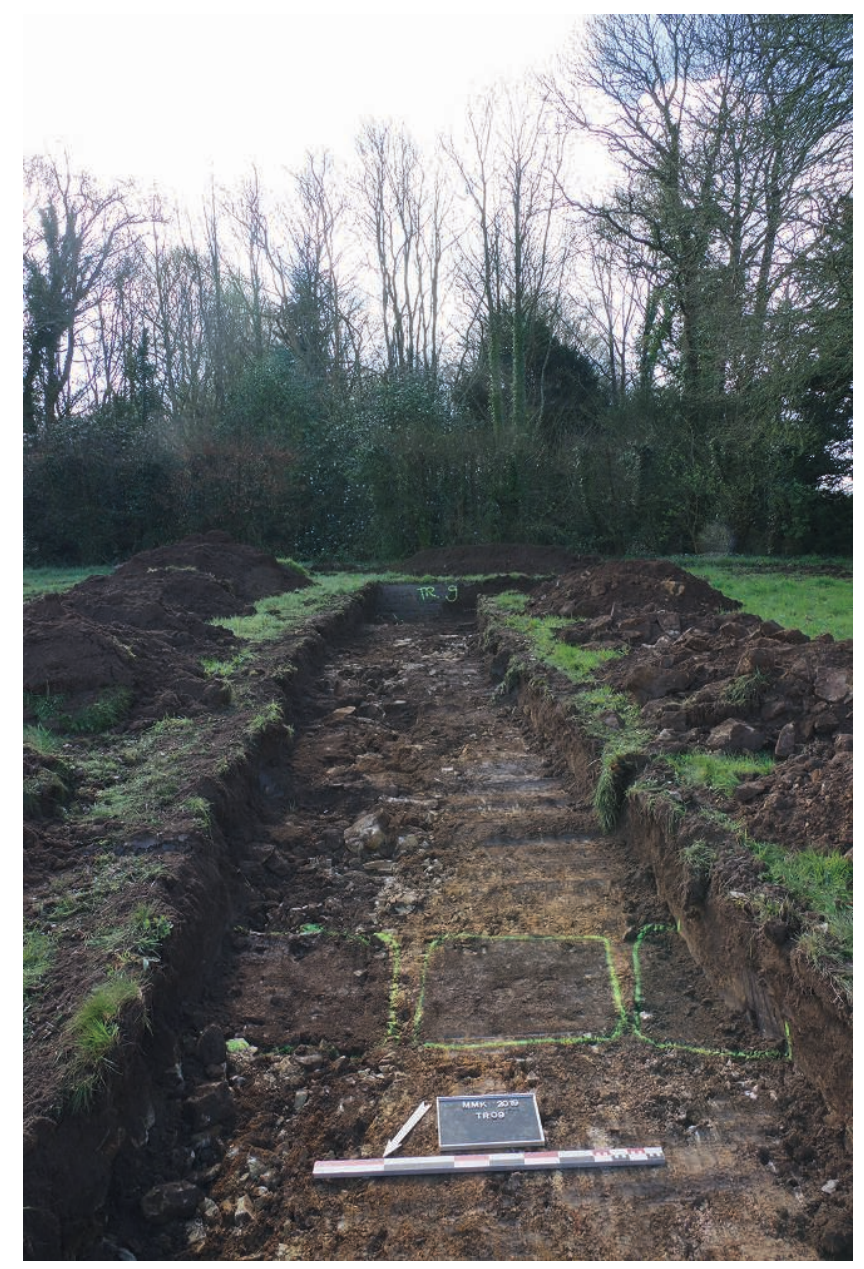

Figure 10 : Vue des fosses de plantation dans la tranchée TR 09 (cliché L. Clouet).

Figure 10: Planting pits in trench TR 09 (photo L. Clouet).

cifiques. La fonction résidentielle se distingue dans l'aménagement d'un jardin d'agrément. Les haies et les bosquets structurent l'espace, organisent le jardin. Les zones potagères n'ont pas nécessairement marqué le sous-sol, bien que nous sachions qu'elles aient été présentes à une époque. Notons que la contemporanéité de l'ensemble de ces vestiges n'est pas évidente. Stéphanie Hurtin rappelle dans le cadre du rapport de fouille des jardins du château de Kerjean à SaintVougay (Finistère) que le jardin est "à considérer comme un ensemble construit qui évolue dans le temps " (Hurtin, 1998, p. 11). Cette phrase souligne la difficulté d'un phasage chronologique précis lorsque l'ensemble des structures archéologiques propres à l'aménagement du jardin suit une certaine homogénéité durant plusieurs siècles.

En outre, bien qu'aménagé et structuré, le jardin du manoir de Kernault ne revêt pas les caractéristiques d'un jardin à la française, mais plutôt celles d'un jardin de la fin du Moyen Âge. Contrairement au premier Moyen Âge, où les extérieurs ont surtout une fonction utilitaire, à partir $\mathrm{du} \mathrm{XI}{ }^{\mathrm{e}}$ s., les jardins « de plaisir " se développent, de plans carrés ou circulaires et toujours clos. Les jardins clos de murs sont par ailleurs souvent ceux des châteaux, palais, maisons fortifiées ou établissements ecclésiastiques (Gesbert, 2003, p. 398-399). En effet, les maisons fortes, bien que rattachées à une réalité économique agricole, sont également associées à des espaces clos et organisés dédiés au confort et au bienêtre du seigneur et de sa famille. Le jardin, signe de raffinement, est depuis l'Antiquité une marque d'appartenance à un monde privilégié (Sirot, 2007, p. 97).

Les études archéologiques régionales de jardins en contexte seigneurial ne sont pas très fréquentes et les éléments de comparaison restent encore rares. Néanmoins, plusieurs sites bretons ont bénéficié de recherches récentes. Au château de Kerjean à Saint-Vougay (Finistère), dans le cadre d'un projet de restauration des jardins, une fouille programmée menée par Stéphanie Hurtin (2000) a eu lieu dans la seconde moitié des années 1990. L'esplanade nord du château a été fouillée à cette occasion. On peut également citer l'opération menée par Cécile Travers dans les jardins du château de Kergroadez, à Brélès (Finistère) en 2012 (Travers, 2012), ou encore celle dirigée par Anne Allimant-Verdillon au château de Coscro à Lignol (Morbihan) en 2002 (Allimant-Verdillon, 2002).

Les premières recherches archéologiques sur le manoir de Kernault permettent de renouveler l'histoire du lieu, de poser de nouvelles questions, de mieux saisir la façon dont étaient traités les abords immédiats du logis et d'alimenter les recherches sur l'archéologie des jardins.

\section{Bibliographie}

Allimant-Verdillon A. (dir.), 2002 - Le jardin du château du Coscro (Lignol, Morbihan). Étude archéologique, rapport final d'opération, Rennes, SRA Bretagne, 343 p.

Brand'Honneur M., 2001 - Manoirs et châteaux dans le comité de Rennes. Habitat à motte et société chevaleresque, Rennes, Presses universitaires de Rennes, 317 p.

Clouet L. (dir.), Kergourlay A., Grall B. (coll.), 2019 - Mellac (Finistère, Bretagne), manoir de Kernault, rapport de diagnostic archéologique, Le Faou-Quimper/Rennes, conseil départemental du Finistère, mission Archéologie/SRA Bretagne, 124 p.

Digou S., Le Gal Y., 2008 - Manoirs et châteaux du Finistère, Quimper, Éditions Palantines, 157 p.

Douard C., 2000 - Manoir de Kernault, Mellac, d'après l'Inventaire général du patrimoine : Dossier IA29000395 réalisé en 2000 par Christel Douard [http://patrimoine.bzh/gertrudediffusion/dossier/manoir-de-kernault-mellac/410eddea-a1a6- 
4d33-b407-afec110a53b0\#historique, consulté le 30 mars 2020].

Douard C., 2002 - Quimperlé et son canton. Images du Patrimoine, Inventaire général des monuments et des richesses artistiques de la France, Rennes, Apogée, 88 p.

Flagelle E., 1878 - " Notes archéologiques sur le département du Finistère ", Bulletin de la Société académique de Brest, 2, IV, p. 1-90.

Gaugain L., Liévaux P., Salamagne A. (dir.), 2019 - La fabrique $d u$ jardin à la Renaissance, Tours, Presses universitaires François-Rabelais, 361 p.

Gesbert E., 2003 - «Les jardins au Moyen Âge : du XI ${ }^{\mathrm{e}}$ au début du $\mathrm{XIV}^{\mathrm{e}}$ s. ", Cahiers de civilisation médiévale, 46, 184, p. 381408.

Hurtin S. (dir.), 1998 - Le château de Kerjean, Saint-Vougay, Esplanade nord, 290271004 H. Archéologie des jardins. Fouille programmée biannuelle, premier rapport intermédiaire, Rennes, SRA Bretagne, $48 \mathrm{p}$.

Hurtin S. (dir.), 2000 - L'esplanade nord du château de Kerjean, Saint-Vougay, 290271004 H. Archéologie des jardins. Fouille programmée biannuelle, rapport de synthèse, Rennes, SRA Bretagne, $159 \mathrm{p}$.

KernÉvez P., 1996 - «Inventaire des mottes, enceintes et châteaux médiévaux du Finistère ", Bulletin de la Société archéologique du Finistère, année 1996, Quimper, Société archéologique du Finistère, p. 115-140.

Kernévez P., 1997 - Les fortifications médiévales du Finistère. Mottes, enceinte et châteaux, Vannes/ Saint-Malo, Institut culturel de Bretagne-Skol-Uhel ar vro/Centre régional d'archéologie d'Alet, 197 p.
Le Calvé J., 1995a - Kernault, la mémoire d'un lieu. L'évolution du rôle du manoir dans le milieu rural $X V^{e}-X X^{e} s$. [inédit; CRBC, Brest, bibliothèque de Kernault; dactylographié], 441 p. + annexes.

Le Calvé J., 1995b - «La seigneurie de Kernault aux Xv et Xvi siècles ", Maner, p. 3-8.

Mignot C., Chatenet M. (dir.), 1993 - Le manoir en Bretagne : 1380-1600, Inventaire général des monuments et richesses artistiques de la France, Paris, Imprimerie nationale, 345 p. (Cahiers de l'Inventaire, 28).

Pla L., Le Rest F., 1991 - Inventaire des archives du manoir de Kernault, fonds de Poulpiquet, mémoire de maîtrise en histoire, Brest, université de Bretagne occidentale, Centre de Recherches Bretonnes et Celtiques [inédit; dactylographié].

Postic F., 1993 - «Kernault, manoir breton : un lieu de mémoire », ArMen, 52, p. 38-49.

Postic F. (dir.), Le Calvé J. (coll.), 1994 - Kernault, manoir breton, Mellac/Quimper, Association de gestion et d'animation du manoir de Kernault/conseil général du Finistère, 32 p.

Sirot E., 2007 - Noble et forte maison. L'habitat seigneurial dans les campagnes médiévales. Du milieu du XII au début du XVI s., Paris, Picard, 207 p.

Travers C., 2012 - Opération menée sur la parcelle nord des jardins du château de Kergroadez (Brélès, 29), Rapport de sondages archéologiques, Lyon/Rennes, Archéoverde/SRA Bretagne, $50 \mathrm{p}$.

WANNER F., 1993 - Inventaire des archives du manoir de Kernault, fonds du Vergier de Kerhorlay, mémoire de maîtrise en histoire, Brest, université de Bretagne occidentale, Centre de Recherches Bretonnes et Celtiques [inédit; dactylographié]. 